William Gallois

\title{
VIOLENCE LEXICALE DE LA CULTURE IMPÉRIALE FRANÇAISE
}

Quel fut le rôle du langage dans la violence de l'impérialisme français moderne?

Prenant l'exemple de la conquête française de l'Algérie des années 1830 et 1840 , je me propose de montrer comment un registre et un langage de la force inédits furent déployés par la France au Maghreb. Bien que châtiments, expéditions punitives et massacres aient été présentés comme autant de réponses indissociablement liées à l'âpreté de l'environnement africain dans lequel les soldats européens opéraient. Ces formes de violence résultèrent, en réalité, d'une série articulée et argumentée d'idées et de politiques véhiculées par les élites de l'Empire à Paris et à Alger. Ces notions se cristallisèrent dans des néologismes. Ces mots n'étaient certes pas porteurs par eux-mêmes de nouveaux modes de violence, mais ils naturalisaient et normalisaient des pratiques de violence et leurs conséquences, dans la mesure où le langage, à moins d'être analysé étymologiquement, est souvent considéré comme sans origine et apparaît comme un miroir du monde. Les néologismes ont le pouvoir de libérer et de redéfinir le réel en répandant dans leur sillage un sentiment partagé de transformation culturelle du monde. Ceux qui, dans les années 1830-1840, propageaient les termes nouveaux dont il va être question ici devaient bien deviner les conséquences pernicieuses d'un tel jeu linguistique.

L'analyse que je propose ici trouve son origine dans la lecture de lettres, rapports, livres et pamphlets rédigés par des soldats, des administrateurs et des politiciens français basés en Algérie entre 1830 et 1840 . On constate que les rédacteurs de ces divers textes 
n'ont emprunté à l'arabe que très peu de mots, et que l'un d'entre eux est passé dans la langue française de manière plus significative que les autres: le terme razzia et sa forme verbale néologique razzier, apparue rapidement, de l'arabe ghāzwa ou incursion. Je vais ici tenter de retracer le parcours de ce vocable depuis l'Arabie préislamique, à travers les formes ibériques médiévales, peu étudiées jusqu'à présent, du castillan, du catalan et du portugais (ainsi que du maltais), dans sa relation avec les premières langues créoles modernes de la Méditerranée (lingua franca, langue franque, sabir), jusqu'à son émergence dans la langue française du XIXe siècle et, de là, dans les langues anglaise, allemande, néerlandaise et d'autres langues modernes européennes.

\section{EMPRUNTS LEXICAUX}

La langue française a, de longue date, emprunté à l'arabe et aux langues du Proche-Orient des termes, souvent d'origine médiévale, qui ont transité par l'italien (sucre et turban), le turc (minaret, café), la Palestine des croisades (gazelle, tasse), l'Ibérie islamique (hasard, amiral) ou, via des filières plus complexes, par l'arabe, le persan et le catalan (aubergine), voire par Tolède et ses grands projets de traduction de l'arabe en latin (alchimie, carafe) ${ }^{1}$.

Le mouvement d'emprunt lexical à l'âge de l'impérialisme moderne fut beaucoup moins sophistiqué que ces migrations anciennes, dans la langue française, de la civilisation orientale avancée qui s'exprimait dans la langue arabe (zénith, algèbre). L'emprunt de termes arabes se concentra au contraire sur la nécessité pratique de décrire la vie (couscous, burnous) et l'environnement auquel les soldats français étaient alors confrontés dans le Maghreb (oued, bled). L'appropriation linguistique du terme razzia leur permit de décrire un aspect du contexte algérien, en particulier des mœurs des peuples des montagnes et du désert.

Les rares linguistes qui ont étudié l'influence de l'arabe sur le

1. H. Wise, The Vocabulary of Modern French : Origins, Structure and Function (London : Routledge, 1997), pp.72-75. Voir également A. Ewert, The French Language (London, Faber and Faber, 1943), pp. 296-97 et A. Dauzat, Précis d'histoire de la langue et du vocabulaire français (Paris, Larousse, 1949), pp. 136-37. 
français au XIXe siècle ont établi un contraste entre le "prestige » des emprunts de l'époque médiévale et le caractère familier ou dialectal de termes issus de l'époque coloniale ${ }^{2}$. Henriette Walter a, par exemple, opposé aux emprunts modernes comme toubib, clebs et kif-kif, les emprunts « à l'arabe scientifique et académique [plus anciens] et plus significatifs ${ }^{3} »$.

L'étymologie du terme arabe ghazwā, dont la racine GH-Z-W est d'origine préislamique, s'avère complexe. Ainsi que l'explique Johnstone dans l'Encyclopédie de l'islam :

Le ghazw (fam. ghaz, pluriel ghizwān) est l'une des institutions les plus anciennes des tribus d'éleveurs de chameaux du nord de la Péninsule arabique, qui ait traversé les siècles sans avoir été transformée par l'islam. Contrairement aux autres pratiques guerrières des Bédouins, notamment la guerre territoriale (manākh) et les expéditions punitives (tha'r /fam. thār), le ghazw visait l'acquisition de chameaux et opérait, en réalité, comme une modalité relativement efficace de redistribution des ressources économiques dans une région où l'équilibre était facilement mis à mal par les catastrophes naturelles ${ }^{4}$.

Ainsi que le montrent son origine et son usage à l'époque moderne, dans certaines régions du monde arabe, le ghazwā décrivait une forme d'incursion liée tant à la destruction qu'à l'équilibre de l'ordre social (d'où la connotation plutôt positive du terme). Et Johnstone de poursuivre :

Les ghazu atténuaient les effets des sécheresses ou des catastrophes naturelles sur le nourrissage des troupeaux de chameaux,

2. J. Chaurand, Introduction à l'histoire du vocabulaire français (Paris, Bordas, 1977), E. Huguet L'Evolution du sens des mots depuis le XVIe siècle (Genève, Droz, 1967), H. Walter et G. Walter, Le Dictionnaire des mots d'origine étrangère (Paris, Larousse, 2009).

3. S. Wright, (éd.), French - an Accommodating language? (Clevedon, Multilingual Matters, 2000); H. Walter, «Le francais, langue d'accueil : chronologie, typologie et dynamique », in S. Wright (éd.), French, pp. 6-30 (16).

4. T.M. Johnstone, «Ghazw». Encyclopaedia of Islam, Second Edition, Edited by P. Bearman, Th. Bianquis, C.E. Bosworth, E. van Donzel, W.P. Heinrichs, Brill Online, 2016. 
seule forme de richesse et de sécurité économique dans la société. Dans la mesure où il visait l'obtention de chameaux, le ghazu, qui était régi par un protocole élaboré, n'occasionnait que peu de violence, les victimes potentielles étant souvent graciées (man $)^{5}$.

La connotation positive des ghazu se retrouve dans les formes qui furent dérivées ultérieurement de la racine GH-Z-W, par exemple dans le terme ghăzi, ou mercenaire, qui désignait les guerriers combattant pour l'islam, au nom de leur foi, aux frontières du monde byzantin ${ }^{6}$. D'autres variantes reflètent également le sentiment de devoir religieux qui pouvait animer la guerre bien que, comme dans le cas du jihā $d^{7}$, l'usage prédominant de la racine et du terme intervînt plutôt dans la description de raids qui visaient les biens ou le bétail de tribus rivales.

Fondamentalement, le ghazwa $\bar{a}$ était une forme de combat très particulière et, par définition, d'envergure limitée. Il était encadré par des principes, voire des lois, et préservait l'ordre social né de la gestion de l'environnement. Comme nous le verrons, bien que le néologisme français ait effectivement intégré la dimension sociétale positive du concept de razzia, c'est finalement sa dimension destructrice qui sembla la plus utile à l'impérialisme français.

Si l'on en croit la littérature, les versions européennes modernes du ghazwā découlent toutes d'une série de termes apparus dans l'Algérie du XIXe siècle : razzia, rezzou, radsja, bazia, razzier. Il faut néanmoins préciser que les premières appropriations « européennes » de l'arabe viennent, en réalité, de l'Ibérie médiévale. Le terme algaza $a^{8}$ apparaît ainsi pour la première fois dans Le Cid en 1140 , et ses variantes et dérivés (comme $g a z i^{9}$ ) dans les langues

5. Ibidem.

6. I. Melikoff, «Ghāzī. » Encyclopaedia of Islam, Second Edition, Edited by P. Bearman, Th. Bianquis, C.E. Bosworth, E. van Donzel, W.P. Heinrichs, Brill Online, 2016.

7. W. Gallois «The Lure of Jihād», in R. Gleave (ed.) Violence in Islamic Thought, vol. 3 (Edinburgh, EUP, 2017).

8. F. Corriente, A Dictionary of Andalusi Arabic (Leiden, Brill, 1997), p. 379.

9. F. Corriente, Diccionario de arabismo y voces (Madrid, Gredos, 1999), p. 333. 
castillane $^{10}$, catalane ${ }^{11}$ et portugaise $\left(\text { gazua }^{12}\right)^{13}$. La signification des termes ibériques correspond bien à celle du terme arabe originel, à savoir une incursion limitée et codifiée ${ }^{14}$.

La forme française razzia apparut pour la première fois en $1840^{15}$ ou en $1841^{16}$ et passa « rapidement dans le langage courant $^{17} »$. Le verbe français razzier, dont seules quelques occurrences textuelles ont été repérées avant 1880, semble avoir mis davantage de temps à être adopté.

Initialement mobilisés afin de décrire le contexte algérien, ces deux termes furent, dès 1848, utilisés en France et en Europe — dans la presse anglaise, par exemple — pour évoquer diverses formes de violence. Ils apparaissaient parfois en italique, pour mettre en évidence leur origine étrangère ${ }^{18}$.

Dictionnaires et ouvrages étymologiques du XIX ${ }^{\mathrm{e}}$ siècle s'accordaient à définir le terme razzia comme un terme emprunté à l'usage algérien de l'arabe classique, et signifiant « incursion militaire $^{19} »$. Cette définition commune perdura au $\mathrm{XX}^{\mathrm{e}}$ siècle, où les idées de saccage, d'incursion et de pillage furent, de manière

10. J. Corominas, Diccionario crítico etimólogico castellano e hispánico, vol. 1 (Madrid, Gredos, 1991), p. 159; F. Corriente, Diccionario arabe-español, $2^{\mathrm{e}}$ éd. (Madrid, Instituto arabe de cultura, 1986).

11. J. Corominas, Diccionario, op. cit., p. 175.

12. R.P.A. Dozt et W.H. Engelmann, Glossaire des mots espagnols et portugais dérivés de l'arabe, $2^{\mathrm{e}}$ édition, (Leiden, Brill, 1869), p. 275.

13. A. Montaner Frutos, «Introducción a la épica de frontera (tradiciones Románica, Bizantino-Eslava e Islámica », in Fuli Papayeorgiu et al. (eds) Ressons èpics en les literatures i el folklore hispànic (Barcelona, ACRINET, 2004), pp. 9-39 (14-16).

14. (http://etimologias.dechile.net/?razia).

15. G.A. Bertrand, Dictionnaire étymologique des mots français venant de l'arabe, du turc et du persan (Paris, L'Harmattan, 2007), p. 121.

16. P. Guiraud, Les Mots étrangers (Paris, PUF, 1965), p. 14.

17. G.A. Bertrand, Dictionnaire étymologique, p. 121.

18. Revue de l'Orient, 7 (1845), p. 154; Revue des Deux Mondes, 10-2 (1851), p. 254; Lettres du Maréchal Saint-Arnaud, vol 2 (Paris, Michel Levy, 1855), p. 67.

19. L.M. Devic, Dictionnaire étymologique des mots français d'origine orientale (arabe, persan, turc, hébreu, malais) (Paris 1876), p. 190. A. Cherbonneau, Dictionnaire arabe-français (langue écrite), vol 2 (Paris, Imprimerie nationale, 1876), p. 794. 
prédominante, associées au terme et à l'histoire de sa migration dans la langue française ${ }^{20}$.

Les études dédiées aux emprunts du français à l'arabe algérien étant rares, il n'est pas inutile de se tourner vers celles qui furent menées, sur le même thème, au Maroc et en Tunisie. Lahcen Amargui s'est interrogé sur la raison pour laquelle «les formes locales du français ont emprunté des mots à l'arabe marocain » ou, plus précisément, sur la manière dont « une langue comme l'arabe marocain est parvenue à enrichir une langue dominante, en dépit de son statut de langue dominée ${ }^{21} »-$ la réponse résidant, d'après Amargui, dans la «nécessité linguistique » évidente de "décrire les réalités spécifiques au monde marocain, inexprimables dans les termes du français de métropole ${ }^{22} »$.

Il est néanmoins légitime de se demander si, dans le cas de l'Algérie de 1850, les soldats français avaient réellement besoin d'un nouveau terme pour décrire leurs opérations au Maghreb. Le registre militaire disposait en effet d'une vaste série de mots pour décrire les diverses formes d'assaut — «attaque », «descente», « rafle », pour n'en citer que trois —, termes utilisés depuis les guerres révolutionnaires et napoléoniennes, tant sur le sol national que hors des frontières.

\section{NOMMER L'INNOMMÉ}

Serait-il possible cependant que l'émergence du terme razzia dans la langue française des années 1840 ait répondu à un besoin de la langue, à savoir désigner ce qui était resté sans nom? Et que cet acte de nomination ait entraîné la cristallisation, la conceptualisation et le développement d'une série de pratiques qui existaient déjà avant la colonisation de l'Algérie? De telles suppositions

20. A. Lanly, Le Français d'Afrique du nord (Paris, PUF, 1962), p. 57 ; Mohammed K. El Bacha, L'Arabe émigré. Dictionnaire des mots français dérivés de l'arabe (Beirut Al'ilmlilmalayin, 1997), p. 468.

21. L. AMARGUI, «Le français du Maroc et l'emprunt à l'arabe », in A. Queffélec, F. Benzakour, Y. Cherrad-Benchefra (éd.), Le Français au Maghreb (Aix-en-Provence, Publications de l'Université de Provence, 1995) pp. 43-52 (43).

22. Ibid., p. 51. 
n'infirment en rien la spécificité de la razzia algérienne; elles tentent d'inscrire la violence dans le contexte des jeux linguistiques de l'époque.

Philip G. Dwyer a montré que le massacre s'est imposé, dans les armées françaises, dès la fin du XVIII siècle et plus particulièrement à l'occasion des guerres des années 1790, comme une arme de guerre conventionnelle ${ }^{23}$. L'idéologie de la prétendue vertu civilisatrice des guerres d'extermination, qui vit le jour à l'époque napoléonienne, entendait justifier les atrocités par des soi-disant bénéfices à long terme et au nom du progrès ${ }^{24}$. Ce système de croyance fut essentiellement appliqué à l'étranger : comme le montre Dwyer, il servit l'expansion coloniale.

Les pratiques de guerre qui eurent cours dans la péninsule Ibérique entre 1808 et 1814, dont Goya a représenté quelques scènes épouvantables - châtiments collectifs, pillages, viols, systématisation des massacres —, rappellent certaines pratiques de la France en Algérie dans les années 1840. Aucun terme ne fut cependant utilisé, ni par les auteurs, ni par leurs victimes, pour « labelliser » les violences commises. Curieusement, l'algaza, qui aurait pu passer dans la langue française, ne le fit point.

Les principaux tropes de la razzia furent établis bien avant 1830, y compris celui qui évoquait le pillage des réserves de nourriture par l'armée française afin de nourrir les vastes armées qui avaient, stratégiquement, été sous-approvisionnées par le commandement politico-militaire à Paris. Les mémoires des généraux comme le maréchal Soult sont, à cet égard, éclairantes. Duc de Dalmatie, ministre de la Guerre et/ou Premier ministre pendant l'essentiel de la période 1830-1847, le maréchal Soult fut l'architecte politique et militaire le plus important de la conquête de l'Algérie. Il avait, à l'instar d'autres stratèges de la campagne algérienne, conduit les troupes de Napoléon dans la péninsule Ibérique; la campagne qu'il mena au Portugal en 1809 est évoquée par Lenoble en ces termes :

23. (https://www.academia.edu/227604/_It_Still_Makes_me_Shudder_ Memories_of_Massacres_and_Atrocities_during_the_Revolutionary_and_ Napoleonic_Wars_).

24. (https://www.academia.edu/6744272/Violence_and_the_revolutionary_and_Napoleonic_wars). 
Quand les habitants fuient, ils échappent aux baïonnettes; et dans la campagne que nous retraçons, ils fuyaient par ordre, par crainte, et pour soustraire leurs denrées [...] à une armée précédée par la réputation de prendre et de faire travailler sans avoir argent pour payer ${ }^{25}$.

Lorsque les populations se levaient contre les forces d'invasion, elles étaient invariablement accusées, sur un ton moralisateur, d'avoir exercé une vengeance injuste à l'encontre des Français, comme, par exemple, dans le cas du meurtre du général Foy qui fut « traîtreusement» et « indignement massacré ${ }^{26}$. La rhétorique de l'armée française mettait souvent en avant l'argument suivant : des populations civiles méritant amplement les «horreurs de la guerre » étaient au contraire traitées avec humanité, ce qui attestait de la haute éthique militaire. De telles allégations pouvaient facilement être contestées par ce qui s'était produit et avait été rapporté, à la même époque, dans d'autres villages où les « habitants », au contraire, avaient été «punis » et leurs maisons «incendiées» pour servir «d'exemple » comme dans le village de Castro-deCaldelas $^{27}$. Les massacres, tristement notoires, de Leyria, Evora et Guarda, par exemple, furent, sans surprise, décrits dans des termes beaucoup plus durs par les généraux anglais, ennemis de l'armée française au Portugal, ainsi par exemple John T. Jones :

[A Evora], la défense fut organisée par le général portugais Leite avec l'aide d'un corps d'armée espagnol; les troupes impériales n'y furent donc confrontées qu'à une résistance armée conventionnelle plutôt qu'à une émeute populaire. Néanmoins, le général Loison livra la ville, tombée le 29 juillet, aux soldats, encourageant plutôt que réprimant le pillage et les atrocités qu'ils y commirent. Ceux qui furent épargnés tressaillent encore lorsqu'ils évoquent la cruauté délibérée et débridée avec laquelle, pendant une journée entière et en toute impunité, femmes et enfants, et prêtres surtout, furent jetés hors de leurs abris, maltraités ou

25. M. Lenoble, Mémoire sur les opérations militaires des Français en Galice, au Portugal et dans la vallée du Tage en 1809, sous le commandement du maréchal Soult, duc de Dalmatie (Paris, Barrois l'aîné, 1821), p. 110.

26. Ibid., p. 179.

27. Ibid., p. 285. 
anéantis. Des témoins crédibles soutiennent que plusieurs milliers de personnes furent tuées et mutilées pendant le massacre. A Guarda, mille deux cents morts furent dénombrés sur le champ de bataille. Nous n'avons aucun détail sur les exactions commises par Loison à Atalya, autre lieu de ses exploits; les habitants y étant peu nombreux, il semble que personne n'en réchappa $[\ldots]^{28}$. »

Nous le verrons, la vertu pédagogique du massacre à des fins d'édification, pour la communication et la représentation du futur - à travers le récit des massacres fait par les survivants deviendra centrale dans la notion de razzia. Ainsi que Jones l'explique, dans cette conception de ce type d'attaque, l'après-coup du raid devait avoir un écho ultérieur. Cette onde de choc reposait sur ce qu'on pourrait appeler une forme d'éthique anticipatrice permettant, par une inversion du sens moral, de présenter des actions particulièrement brutales comme un bien supérieur; le tout selon une logique sous-jacente du mal nécessaire, explicite seulement pour ses partisans, mais qui se révèlerait finalement à tous.

\section{L'HISTOIRE SECRÈTE DE LA RAZZIA}

La question de la moralité des actes de guerre anima les débats dans la colonie algérienne à propos de la razzia, avant même l'apparition du terme dans la langue française. Les premières mentions du terme dans les documents militaires identifièrent généralement la razzia comme une pratique locale - telle la razzia lancée par le Bey de Constantine contre une tribu rivale en août $1833^{29}$, alors que dans le même temps, dès le mois d'octobre de la même année, un général français, Schauenbourg, entreprenait lui-même une

28. J. Tones, Account of the war in Spain and Portugal, and in the south of France, from 1808 to 1814 inclusive (London, T. Egerton, 1821), p. 23.

29. Service historique de la Défense, Vincennes (SHD) 1 H 21. J. Nicot, et P. Carre, Algérie, 1830-1945 : inventaire de la série H : soussérie 1 H 1-1090. Tome II, 1 H 1 à 93 : 1830-1843 : inventaire analytique et index (Vincennes: Service historique de l'armée de terre, 2002). Sources anonymes et parfois datées, empruntées à Nicot et Carre. 
razzia contre une faction hostile de la tribu des El Krachna ${ }^{30}$. Cette appropriation de la pratique était méprisée par une partie des élites politico-militaires aux commandes de l'Algérie à la fin des années 1830, notamment par le gouverneur général Valée (1837-1840) qui avait annoncé son intention d'abolir ladite pratique dès $1838^{31}$. Dans sa correspondance, en date de novembre 1840, il évoque le débat moral opposant les « légalistes », dont il était et qui jugeaient l'imitation de la razzia peu flatteuse pour la civilisation française, et les réalistes conduits par le maréchal Soult et le maréchal Bugeaud (gouverneur général entre 1841 et 1847) qui la jugeaient, au contraire, appropriée et nécessaire dans le contexte algérien de l'époque, et pour qui c'était là même « la véritable guerre qu'il faut faire aux Arabes ». Pour Valée,

[i]l faut accepter l'Algérie avec toutes les mauvaises chances qu'elle présente, l'influence du climat met dans les hôpitaux une grande partie de l'armée, la construction de bâtiments militaires, l'envoi de lits pour tous les soldats et une nourriture abondante et [...] de bonne qualité sont les seuls moyens d'améliorer cette situation [...], je suis loin de penser que la guerre puisse se terminer promptement, on ne peut soumettre un peuple en quelques jours, [mon] système vaut bien celui de courses et de razzias sans but ${ }^{32}$.

Les « réalistes », quant à eux, mêlaient pragmatisme et idéalisme selon la logique vraisemblablement acquise lors des campagnes répressives de la Révolution et des guerres napoléoniennes ${ }^{33}$. La razzia leur apparaissait réaliste également en un autre sens : c'était un moyen d'expédier les opérations militaires alors que les coûts de la conquête d'Algérie avaient excédé les estimations initiales et, en conséquence, entraîné un mécontentement en métropole. Le recours à la razzia était aussi idéologique en ce qu'il véhiculait une série d'opinions sur les croyances dites « locales », les formes de langage « compréhensibles par les indigènes » et les qualités prétendument révolutionnaires de certaines formes de violence.

Ces débats se déroulèrent dans la correspondance privée et les

30. SHD 1 H $22-1$ (11 octobre).

31. SHD 1 H $55-3$ (12 avril 1838).

32. SHD 1 H $73-1$ (2 novembre 1840$)$.

33. SHD 1 H $72-2$ (26 octobre 1840). 
discours publics des militaires et des élites politiques de Paris et d'Alger avant que le terme razzia eût officiellement migré du parler ordinaire des soldats français d'Afrique du Nord (lesquels ne laissèrent que peu de traces documentaires) vers la langue française. On peut légitimement se demander si la pratique de la razzia fut finalement une réponse militaire subalterne au contexte algérien de l'époque ou une théorie du conflit développée par les élites métropolitaines. Le fait que partisans et détracteurs de la razzia définirent semblablement celle-ci comme une stratégie de guerre dès le début des années 1840 suggère qu'il ne s'agissait pas là d'un infléchissement spontané des pratiques (correspondant à une évolution empirique du langage sur le terrain) mais au contraire d'un langage imposé dans ce domaine militaire et central dans la gouvernementalité de la conquête.

En novembre 1841, Bugeaud chargea le général Changarnier de mettre en œuvre cette stratégie de razzias et d'embuscades à partir de Blida ${ }^{34}$, alors même que la presse anglaise s'emparait du terme pour stigmatiser l'impérialisme français et son «abject système de razzia à Alger ${ }^{35} »$. Ce genre de commentaire n'était pas rare outre-Manche; il montre que les razzias françaises y étaient d'emblée comprises comme une forme extrême de violence, plus virulente que le brigandage arabe, et infligée aux Algériens comme une sorte de leçon de civilisation sous la forme de l'affirmation de la puissance. La correspondance privée de personnalités comme le maréchal Soult met au jour cette croyance française en la prétendue portée pédagogique de la razzia : « la terrible leçon que [les tribus] ont reçue », écrit-il à propos de la razzia d'Erdough, « les a mises moralement à notre discrétion $»^{36}$.

$\mathrm{Du}$ point de vue sociolinguistique, il est clair que le terme razzia a sensiblement évolué sur une courte période. En termes de classification linguistique, il fit son apparition dans la langue française, dès les premiers jours de la conquête algérienne, sous la

34. SHD 1 H $78-1$ (24 novembre 1841).

35. «Les Français sont en passe de récolter les fruits de leur abject système de razzia à Alger. Ils ont donné un exemple aux Arabes que ces derniers, malgré leur talent légendaire pour le pillage, n'avaient pas encore imaginé et qu'ils allaient apprendre de la civilisation des envahisseurs européens », The Ipswich Journal, 8 october 1842, Issue 5399.

36. SHD 1 H $89-3$. 
forme d'un calque : une tentative de "substitution complète d'un morphème local » qui était, en réalité, une forme d'importation plutôt que de substitution, au sens d'une appropriation de cette pratique locale ${ }^{37}$, et ce tant du point de vue sémantique que phonétique : dans son utilisation française, le terme razzia sonnait comme l'original en arabe et renvoyait à ce qui avait été compris de la pratique qu'il évoquait. La phase d'emprunt, en tant que telle, s'étendit de 1830 à 1838 environ, époque à laquelle apparut progressivement « un chevauchement sémantique entre le morphème d'origine et son appropriation française, qui lui [ajouta] une couche sémantique nouvelle ${ }^{38} »$, prenant ainsi la forme d'un synonyme d'emprunt. Cette seconde phase engendra, chez les utilisateurs français du terme, défenseurs comme pourfendeurs de la pratique, une utilisation toujours plus radicale, violente et éloignée du terme d'origine. En 1840, ce glissement sémantique — au sens du « processus par lequel des termes locaux sont appliqués à des phénomènes culturels nouveaux, relativement similaires à certains aspects de la culture d'origine ${ }^{39} \gg$ — était pratiquement achevé : la razzia, dans son acception française, exprimait désormais une forme de violence, en vérité complètement inédite dans le Maghreb, mais dont d'aucuns persistaient à dire qu'elle était fondée sur des archétypes locaux.

Quant à savoir si l'on peut effectivement caractériser la phase finale de l'évolution du terme de confusion sémantique — «dans laquelle les nuances d'origine sont occultées par l'incidence d'une synonymie partielle entre deux langues ${ }^{40} »-$, les points de vue divergent selon les destinataires. Si nombre de Français étaient sincèrement convaincus de la fidélité à l'original de leur version de la razzia, pour les victimes algériennes de la violence impériale - notamment celles qui avaient été habituées à certaines formes antérieures de pression coloniale ottomane (comme celle des colonnes militaires envoyées dans les régions montagneuses pour y collecter l'impôt) —, c'était bien plutôt la « confusion » et la sidé-

37. E. Haugen, « The Analysis of Linguistic Borrowing », Language 26-2 (1950), pp. 210-231 (219, 212).

38. E. Haugen, «The Analysis of Linguistic Borrowing », Language vol. 26, no. 2 (avril-juin, 1950), pp. 210-231 (219).

39. Ibid, p. 219.

40. Ibidem. 
ration qui étaient à l'œuvre quand les escouades de soldats français déployèrent avec détermination à leur encontre une violence annihilatrice, et ce dès leurs premiers contacts politiques avec ces tribus, jugées « récalcitrantes ». Les écrivains français du XIXe siècle définirent les razzias de leurs compatriotes comme la continuation de normes culturelles préexistantes - on se référera par exemple, à cet égard, à la définition du Grand Dictionnaire Larousse selon lequel « on employait les razzias du temps du gouvernement turc; on pillait les tribus qui refusaient de payer l'impôt et on les forçait ainsi à se soumettre [...] Nous avons imité les Turcs ${ }^{41} »$. Les auteurs locaux, en revanche, tels que Hamdan Khodja ou Ahmed Bey, de Constantine, proposèrent une tout autre lecture des événements qui mettait en lumière les horreurs sans précédent de la violence impériale moderne, « une injustice dont l'histoire des oppresseurs ne nous a jamais donné d'exemple », selon la formule du Bey ${ }^{42}$.

L'Afrique du Nord, comme bien d'autres régions, avait connu diverses formes de domination politique et d'assujettissement; mais la razzia française se distingua par la rapidité avec laquelle elle se développa et dériva de sa racine sémantique originelle pour incarner de nouvelles cultures et pratiques de la violence. Du point de vue linguistique, l'idée, défendue par Haugen, que l'emprunt linguistique tient davantage du vol que de l'emprunt lexical proprement dit apparaît ici dans toute sa pertinence, mais Haugen n'a pu imaginer comment l'emprunt permanent — un vol — n'est pas seulement linguistique. La perte définitive de biens, fut bien la conséquence de ce processus pour les victimes de cette version coloniale de la razzia. Ces pratiques ne furent pas certes inventées en Algérie mais préfigurées lors des conquêtes napoléoniennes, lors desquelles elles étaient étrangement restées sans nom.

Ce vide linguistique fut comblé par la nouvelle razzia à la française avec une rapidité telle que, dès le début des années 1840 , elle désigna, dans l'usage commun des langues française, algérienne et européennes, un projet de violence destructrice qui visait à assurer le contrôle total d'un territoire et de son peuple. Le processus de

41. Larousse, Grand Dictionnaire du XIXe siècle, vol. 13 (Paris, Larousse, 1875), p. 748.

42. A. Temimi, Le Beylik de Constantine et Hadj 'Ahmed Bey (1830-1837) (Tunis, Publications de la Revue d'histoire maghrébine, 1978), p. 228. 
transformation linguistique prit moins d'une décennie, balayant des siècles d'évolution sémantique progressive de sa racine GH-Z-W qui s'était faite via des extensions de sens limitées façonnant un air de famille à travers une vaste extension géographique.

Pour les architectes de la conquête et de la construction de l'Algérie française, la razzia devint une sorte de cryptogramme doté de pouvoirs magiques. Comme l'écrivit un général, elle était «le seul moyen que nous ayons à notre disposition ${ }^{43}$ »; pourtant cet usage stratégique de la razzia servit aussi à masquer les changements rapides dans les pratiques. En 1838, le terme évoquait un raid punitif contre un opposant qui avait refusé de coopérer, raid ayant pour but de lui dérober de grandes quantités de nourriture et du bétail et éventuellement de prendre quelques otages; quatre ans plus tard, elle signifiait un raid au cours duquel les populations civiles des tribus rebelles étaient massacrées, les quelques survivants n'étant épargnés que pour qu'ils puissent témoigner des atrocités et de la cruauté dont les forces coloniales françaises étaient capables afin d'imposer leur domination. Alors qu'en 1838, les razzias françaises visaient surtout les ressources convoitées par une armée sous-approvisionnée, dès 1842 elles avaient pour objectif la destruction des cultures, des réserves de céréales et des vergers afin de capturer, économiquement et socialement, les Algériens restés sur leurs terres. Ces agissements en rappellent d'autres qui se produisirent dans d'autres colonies de peuplement à la même période, en Australie, en Nouvelle-Zélande et en Amérique, bien que les travaux universitaires sur l'Algérie soient curieusement peu enclins à accepter cette réalité et ces rapprochements ${ }^{44}$.

Pour les Français de cette colonie de peuplement initiale, la razzia devint totémique. Le mot fit-il l'acte? Peut-être pas. Reste que le logos ne peut être séparé de la praxis, dans la mesure où ce fut le premier qui systématisa la seconde par l'intégration d'une série évolutive de comportements, en rompant avec le sens originel du terme et en l'exposant, dans la langue française, à une série de mutations.

43. SHD 1 H $90-2$.

44. Voir, en particulier M.C. Thoral, « French Colonial CounterInsurgency : General Bugeaud and the Conquest of Algeria, 1840-47 », British Journal for Military History 1-2 (2015), pp. 8-27 et Th. Rid, «Razzia: A Turning Point in Modern Strategy », Terrorism and Political Violence (2009), 21-4, pp. 617-35. 
La forme néologique de la razzia représente un moment inédit d'emprunt culturel impérial, qui a peu retenu l'attention des linguistes, car le terme emprunté n'est pas assez prestigieux. Pourtant ce mouvement lexical est sans doute plus intéressant que la migration des termes arabes plus sophistiqués dans les cultures européennes au Moyen-Age. Comme Attewell et d'autres historiens du colonialisme du XVIII ${ }^{e}$ siècle l'ont montré, les Européens étaient alors spontanément enclins à accepter l'idée d'emprunter des concepts et des savoirs considérés comme supérieurs venus du monde colonisé ${ }^{45}$. A partir des années 1830, cependant, l'appropriation de la razzia témoigne d'un nouveau type de transfert, bien plus pernicieux, qui par un funeste effet de miroir permet aux Européens de mimer des formes de violences et de «barbarisme », en intégrant un nouveau lexique d'emprunt dans les langues européennes $^{46}$. Ce n'est pas un hasard si Hamdam Khodha, en 1833, intitula sa critique des terribles réalités de l'impérialisme en Algérie Le Miroir, pointant la trompeuse « mimesis » d'une invasion menée au nom de la civilisation mais fondée sur la terreur ${ }^{47}$.

\section{William GALLOIS \\ Traduit de l'anglais par Anne Wilhelmi}

45. G. Attewell, «Yunani Tibb and Foundationalism in Early Twentieth-Century India: Humoral Paradigms between Critique and Concordance », in P. Horden, E. Hsu (ed), The Body in Balance : Humoral Medicines in Practice, Epistemologies of Healing; 13, Berghahn Books, Oxford, 2013, pp. 129-148.

46. H. Khodja, Le Miroir : aperçu historique et statistique sur la Régence d'Alger, Paris, Sindbad, 1985 [1833].

47. Au moment de conclure, il me faut remercier, pour les conseils qu'ils m'ont prodigués, en personne ou à travers leurs travaux, lors de la rédaction de ce texte, Ilan Pappé, Olivier Le Cour Grandmaison, Ann Thomson, Steve Nutt, Ann Laura Stoler, Michael Taussig, Robert Young, Hosni Kitouni, Jonathan Wyrtzen, Dionisius Agius, Steven Hutchinson, Adam Watt, Zoe Boughton, Aidan Coveney et Gavin Murray-Miller. Les lecteurs passionnés souhaiteront peut-être également se référer à mon ouvrage A History of Violence in the Early Algerian Colony (London, Palgrave Macmillan, 2013), ainsi qu'à mon article intitulé « Genocide in nineteenth-century Algeria », Journal of Genocide Studies, 15-1 (2013), pp. 69-88. 